\title{
Heterogeneous nucleation and self-nucleation of isotactic polypropylene micro-droplets in immiscible blends: from nucleation to growth-dominated crystallization
}

\author{
Bao Wang ${ }^{\mathrm{a}}$, Roberto Utzeri ${ }^{\mathrm{b}}$, Maila Castellano ${ }^{\mathrm{a}}$, Paola Stagnaro ${ }^{\mathrm{b}}$,
}

Alejandro J. Müller ${ }^{\mathrm{c}, \mathrm{d}, *}$, Dario Cavallo ${ }^{\mathrm{a}, *}$

${ }^{a}$ Department of Chemistry and Industrial Chemistry, University of Genoa, Via Dodecaneso 31, 16146, Genova (Italy)

${ }^{b}$ Institute for Chemical Sciences and Technologies (SCITEC), CNR, Via De Marini 6, 16149 Genova, Italy

${ }^{c}$ POLYMAT and Polymer Science and Technology Department, Faculty of Chemistry, University of the Basque Country UPV/EHU, Paseo Manuel de Lardizábal, 3, 20018 Donostia - San Sebastián, Spain

dIKERBASQUE, Basque Foundation for Science, Bilbao, Spain

*corresponding authors:

dario.cavallo@unige.it; alejandrojesus.muller@ehu.es 


\section{Supporting Information}

(a)
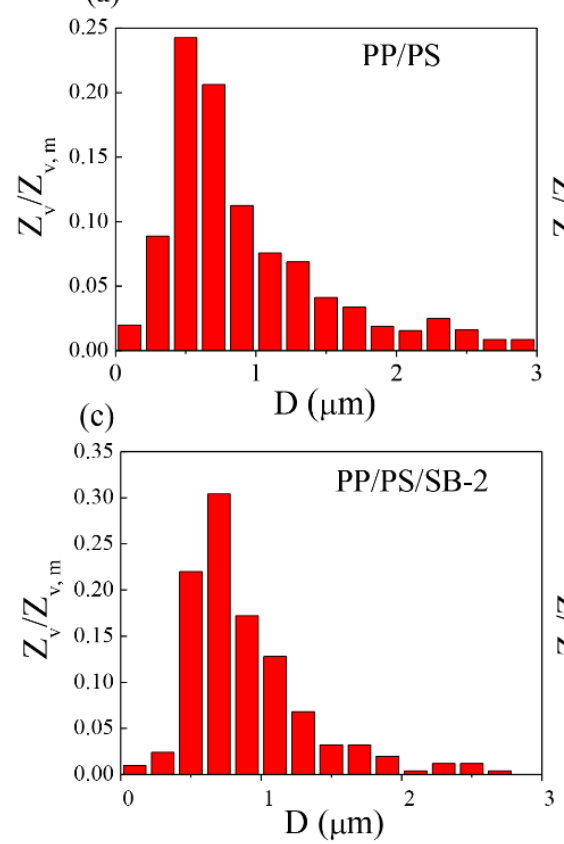

(b)
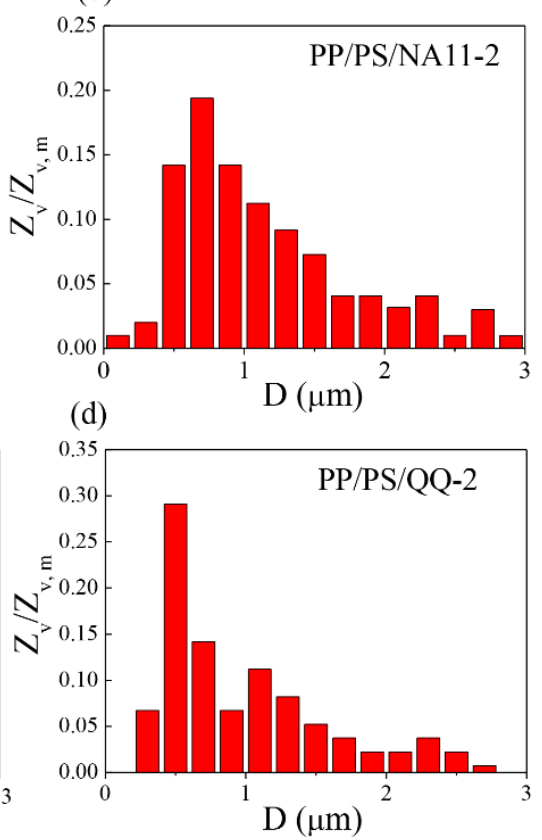

Figure S1. Droplet size distribution of prepared blends, more than 200 droplets are calculated for each blend.

(a)

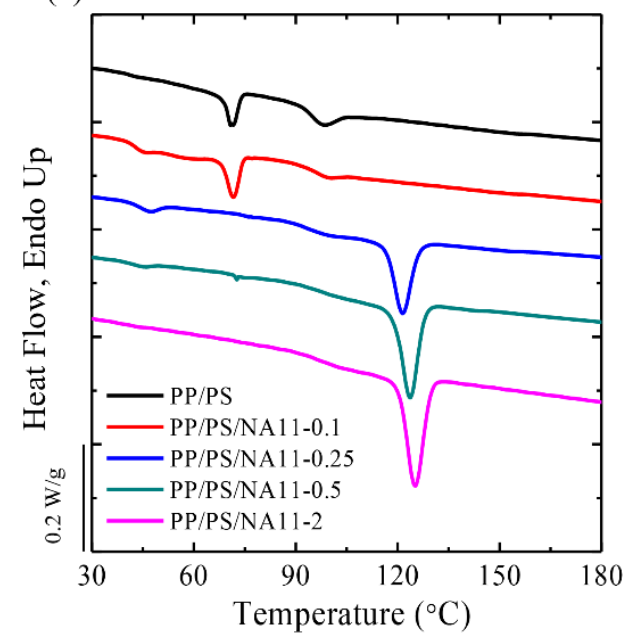

(b)

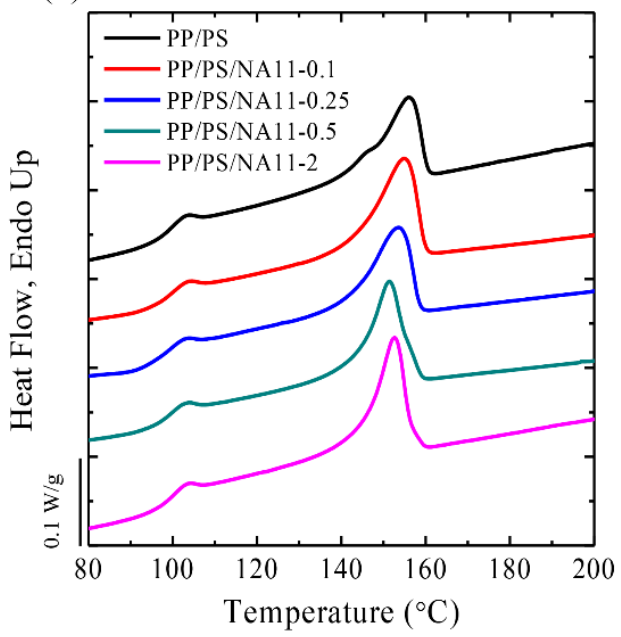

Figure S2. Cooling (a) and heating (b) curves of the PP/PS/NA11 blends with different concentrations of NA11. 


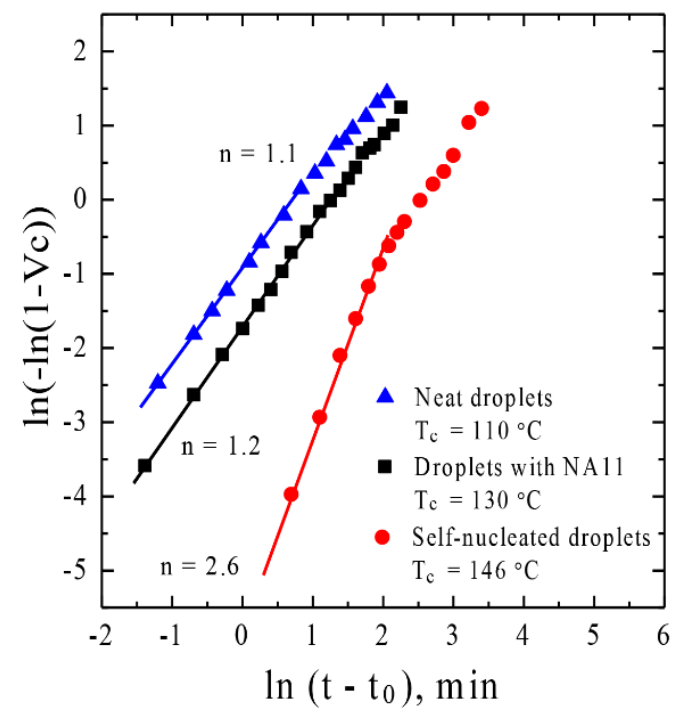

Figure S3. Avrami plot of the crystallization data related to: PP/PS/NA11-0.25; self-nucleated i-PP droplets from $T_{s} 160{ }^{\circ} \mathrm{C}$ (Domain II); (c) self-nucleated i-PP droplets from $T_{s} 210{ }^{\circ} \mathrm{C}$ (Domain I). The colored lines represent linear fittings in the conversion range 3-20\%, from which the indicated values of Avrami index are obtained.

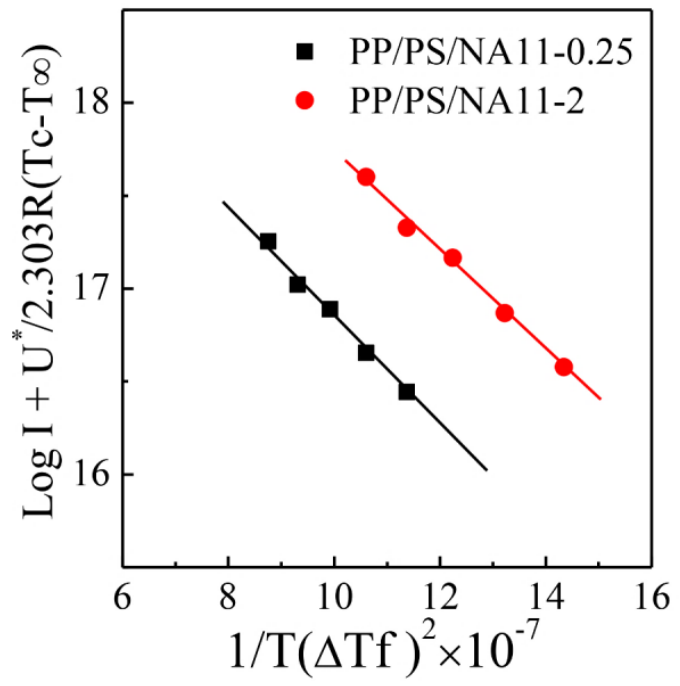

Figure S4. Nucleation rate as a function of temperature for PP/PS/NA11-2 and PP/PS/NA11-0.25 blends according to heterogeneous nucleation theory. 